\title{
位相非感受型光コヒーレンス血管造影
}

\section{Phase-insensitive Optical Coherence Angiography}

\author{
O洪暎周 ${ }^{1,2)}$, 巻田修一 ${ }^{1)}$, 山成正宏 ${ }^{1)}$, 三浦雅博 ${ }^{1,3)}$, 金秀鉉 ${ }^{2)}$, 谷田貝豊彦 ${ }^{1)}$, 安野嘉晃 ${ }^{1)}$ \\ ${ }^{\circ}$ Youngjoo Hong ${ }^{1,2)}$, Shuichi Makita ${ }^{1)}$, Masahiro Yamanari ${ }^{1)}$, Masahiro Miura ${ }^{1,3)}$, Soohyun \\ $\mathrm{Kim}^{2}{ }^{2}$,Toyohiko Yatagai ${ }^{1}$, and Yoshiaki Yasuno ${ }^{1)}$ \\ $\mathrm{COG}^{1)}$, 韓国科学技術院 ${ }^{2}$, 東京医科大学 ${ }^{3)}$ \\ Computational Optics Group in the University of Tsukuba ${ }^{1)}$, Korea Advanced Institute of Science \\ and Technology ${ }^{2}$, Department of Ophthalmology, Tokyo Medical University ${ }^{3)}$ \\ E-mail: hongyoungjoo@kaist.ac.kr
}

\begin{abstract}
A non-invasive, phase-insensitive optical coherence angiography method has been demonstrated for in vivo human retina based on high-speed spectral-domain optical coherence tomography at $840 \mathrm{~nm}$ center-wavelength. Three-dimensional choroidal vasculature was obtained by segmenting choroidal vessels by using threshold values from the intensity distribution in each depth plane relative to the retinal pigment epithelium layer. A vascular projection image was obtained by integrating the segmented choroidal vasculature. The segmented vascular image was compared with the images obtained using existing invasive methods such as indocyanine green angiography (ICGA), to check the feasibility of the alternative method.
\end{abstract}

\section{Introduction}

Examination of ocular vascular integrity is important for the diagnosis of ophthalmic diseases, because the eye receives its nutrient supply through the ocular vessels. Fluorescein angiography (FA) and indocyanine green angiography (ICGA) are frequently used methods for ocular vessel imaging. Although it is possible to obtain satisfying images of the vasculature with FA and ICGA methods, there are some adverse allergic reactions due to the injection of the dye. These reactions range from skin rash and itching, which are mild, to rare but severe reactions like anaphylaxis, which may prove to be life threatening. Therefore, one of the requirements for non-invasive ocular angiography is to avoid allergic reactions.

Optical coherence tomography (OCT) is a low-coherence interferometer-based noninvasive medical imaging method that can provide high-resolution, sectional images of biological tissues. The recently developed Fourier domain (FD) OCT makes it possible to obtain high resolution images with high speed. Additionally, the volume flow dynamics characteristic of blood vessels can be obtained by Doppler OCT. Blood flow can be measured from the phase change between adjacent A-lines [1]. However, to measure the phase change, dense scanning between A-lines is necessary with phase-sensitive technique. The dense scanning is expensive for measurement time, especially for three-dimensional measurement, and it is difficult for some patients to endure a long measurement time and to avoid eye movements during the measurement.

Therefore we developed a phase-insensitive optical coherence angiography (OCA) method using light absorption and the scattering property of blood for choroidal vessel segmentation based on spectral domain (SD) OCT. Three-dimensional choroidal vasculature and twodimensional images of similar quality to that of ICGA are obtained.

\section{System}

A standard SD-OCT system is employed to obtain threedimensional retinal OCT images. A super-luminescent diode (SLD) which has a center wavelength of $840 \mathrm{~nm}$ and a FWHM spectral bandwidth of $50 \mathrm{~nm}$ was used as a light source. Polarization controllers were used after the SLD, and before the reference and sample arms. An optical isolator was used to prevent re-incident light from entering the source. A 20/80 fiber coupler was used to split the beam; $80 \%$ for the reference arm and $20 \%$ for the sample arm. A 78D lens was used to focus on the posterior of the human eye and the measured axial resolution was 6.4um in the tissue. The optical probing power was $700 \mathrm{uW}$. A transparent diffractive grating of 1200 lines $/ \mathrm{mm}$ was used to separate the coherent light in the spectrometer. $18.7 \mathrm{kHz}$ line scan rate CCD was used for the detection; the exposure time of one A-line was 53.1 us. The sensitivity of this system was measured to be $99.3 \mathrm{~dB}$ at a $100 \mathrm{um}$ depth and $92.9 \mathrm{~dB}$ at a $2 \mathrm{~mm}$ depth.

\section{Phase-insensitive optical coherence angiography}

A three-dimensional (3D) human retinal structure could be obtained with high-speed SD-OCT. Fig. 1 describes the process of choroidal vessel segmentation. First, the anterior boundary of the retina was determined by an algorithm similar to that of Mujat et al [2], but excluding the snake algorithm. Next, a highly reflective layer, the retinal pigment epithelium (RPE) and the choriocapillaris, were determined as the maximum gradient position lower than $122.5 \mathrm{um}$ (25 pixels) from the anterior boundary. In our method, the position of RPE is used as a reference depth layer.

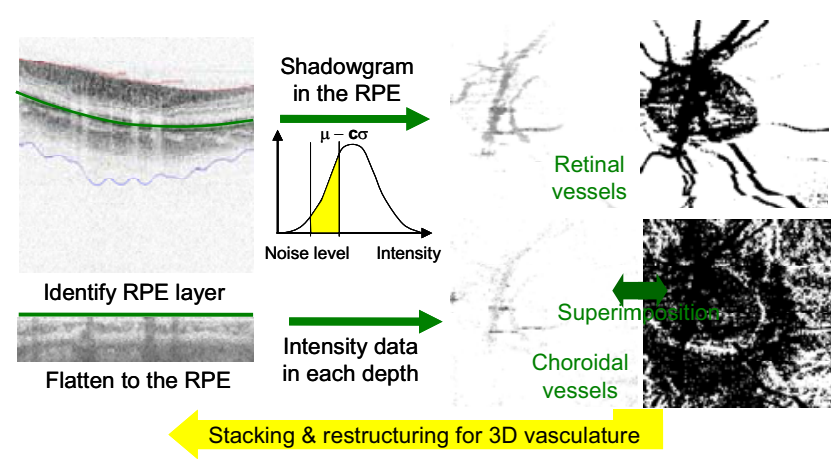

Fig. 1. Process of choroidal vessel segmentation 